\title{
Gestational diabetes: False dichotomy and slippery slope
}

\author{
K.S. Joseph ${ }^{1}$ and Sid John ${ }^{1}$ \\ ${ }^{1}$ University of British Columbia
}

September 25, 2021

\section{Gestational diabetes: False dichotomy and slippery slope} Sid John, ${ }^{\text {a K S Josepha,b }}$

${ }^{a}$ Department of Obstetrics and Gynaecology, University of British Columbia, Vancouver, British Columbia, Canada. ${ }^{\mathrm{b}}$ School of Population and Public Health, University of British Columbia, Vancouver, British Columbia, Canada.

Linked article: This is a mini commentary on PWG Tennant et al., pp. xxx-xxx in this issue. To view this article visit http://dx.doi.org/ $\mathrm{xxxxx}$

Word count: 542

Disclosure of interests: None declared.

Tennant et al used an elegant non-experimental approach to assess the effects of fasting plasma glucose (FPG) and gestational diabetes mellitus (GDM) diagnosis on GDM complications. Their study, based on data from 2 hospitals in the UK, highlights inconsistencies in current GDM diagnosis and management by showing that GDM complications are more common among women with a lower FPG who are not diagnosed with GDM (compared with women with a higher FPG who are diagnosed with GDM). Thus, a woman with an FPG of $5.5 \mathrm{mmol} / \mathrm{L}$, who is not diagnosed with GDM, had a $32 \%$ risk of delivering a large-for-gestational age (LGA) infant, while a woman with an FPG of $5.6 \mathrm{mmol} / \mathrm{L}$, who is diagnosed with GDM, has an LGA risk of $15 \%$.

Diagnosis and intervention based on dichotomizing a biological parameter that shows a linear relationship with adverse events can be fraught with problems, especially when the events cluster around the parameter mean. Lowering the FPG diagnostic threshold to [?]5.1 mmol/L would potentially benefit a fraction of women with LGA infants (105 additional cases in Tennant et al's study) but not the majority of LGA cases that would occur among women with an FPG $<5.1 \mathrm{mmol} / \mathrm{L}$ (852 cases; Tennant et al. Table 1$)$. Similarly, such lowering of the FPG threshold would potentially benefit 11 cases of shoulder dystocia but not the 68 cases among women with an FPG $<5.1 \mathrm{mmol} / \mathrm{L}$. Tennant et al's study also highlights the slippery slope presented by dichotomization: if the diagnostic threshold for GDM is lowered to [?]5.1 mmol/L, women with an FPG of $5.0 \mathrm{mmol} / \mathrm{L}$ who are not diagnosed with GDM, would have a $22 \%$ risk of LGA, while women with an FPG of $5.1 \mathrm{mmol} / \mathrm{L}$ who are diagnosed with GDM, would have a $9 \%$ risk of LGA (Tennant et al. Supplementary Table 3).

The linear relation between plasma glucose and GDM complications, and the dichotomized approach to diagnosis and management, also raise some ethical and legal questions. If there is little difference in the risk of GDM complications between women marginally below and those at and marginally above the diagnostic threshold, is it defensible to label and manage such women differently?

Although Tennant et al argue for a reconsideration of international GDM diagnosis guidelines, they also advocate a graded approach for dealing with hyperglycemia: lower levels of plasma glucose warrant less 
concern and a lower intensity of intervention than higher levels. Concerns and intervention would need to be appropriately modified by maternal factors and other clinical inputs (e.g., fetal biometry). Such a graded approach (with disease severity viewed on a spectrum) has long been a part of medical theory and practice. On the other hand, there also exists a strong desire among both physicians and patients for a simpler framework, which favours the dichotomization and labeling approach to diagnosis and management.

Although hyperglycemia is an important causal factor for LGA, shoulder dystocia, and perinatal death, each of these complications is a heterogenous entity with a multi-factorial etiology. Appropriate diagnosis and treatment of hyperglycemia will address a fraction of such complications, while a comprehensive attempt to prevent these complications will require a multi-faceted strategy of fetal surveillance, diagnosis, treatment and obstetric intervention. Defining gestational diabetes using a hyperglycemic threshold is a seductive proposition that represents a false dichotomy, and hyperglycemia is perhaps better addressed clinically using a graded approach. 